\title{
Presencia de cepas diarreogénicas de Escherichia coli y estudio de genes de virulencia en aislados desde fecas de dos poblaciones de lobo marino común, Otaria flavescens en el norte de Chile
}

\author{
Presence of diarrheagenic strains of Escherichia coli and virulence genes study in isolates from \\ feces of two populations of South American sea lion, Otaria flavescens in Northern Chile \\ Paula Salinas C. ${ }^{1}$, Rubén Moraga M. ${ }^{1}$, Edgardo Santander . $^{1}$ y Walter Sielfeld K. ${ }^{1}$ \\ ${ }^{1}$ Departamento de Ciencias del Mar, Universidad Arturo Prat, Casilla 121, Iquique, Chile \\ rmoraga@unap.cl
}

\begin{abstract}
This research analyzed the effect of fecal contamination caused by sewage, and its relationship with the presence of enteropathogenic strains of Escherichia coli in the feces of South American sea lions (Otaria flavescens) from Iquique Bay and surrounding waters. The E. coli strains isolated from the water column of the bay and sewage outlet, showed the presence of the gene eae. In the strains isolated from the
\end{abstract}

\section{Introducción}

La descarga de aguas residuales urbanas en el mar afecta su calidad, producto de la gran variedad de microorganismos presentes en ellas (Cloete \& Muyima 2001). El ingreso de microorganismos patógenos al cuerpo de agua receptor ocasiona un riesgo para la salud pública y el ecosistema, ya que estos microorganismos pueden afectar a rutas migratorias de peces, ballenas y bancos de moluscos y crustáceos, colonias reproductivas de aves y lobos marinos (Acevedo et al. 2003). Estos pueden ser afectados por bacterias de origen entérico como Salmonella, Vibrio cholerae, Shigella, Campylobacter, Yersinia, Listeria, Clostridium y cepas patógenas de Escherichia coli (Araya 2003). La información sobre enfermedades producidas por bacterias entéricas en mamíferos marinos es escasa, la cual principalmente se ha estudiado en animales domésticos y de producción como el ganado ovino, bovino y porcino (Romero \& Rodríguez 1983, Nataro \& Kaper 1998, García et al. 2002). Por otro lado, el conocimiento de los tipos de bacterias en animales es de importancia sanitaria ya que existe evidencia que algunas cepas patógenas en humanos han sido aisladas de animales salvajes como ECEH en ciervos y UPEC en avifauna salvaje (Blanco et al. 2001, García et al. 2002).

Actualmente, los estudios sobre patologías que afectan a los mamíferos marinos se han incrementado en feces of both sea lion colonies no presence of virulent diarrheagenic genes was detected. The genotyping of strains resulted in clusters according to their origin and showed separation of the samples of $E$. coli population of the colonies of sea lions and those of the strains isolated from the column water of Iquique Bay.

Key words: Enteropathogenic, fecal contamination, Iquique Bay

Norteamérica y Europa (Ceballos et al. 2005, Ceballos \& Erlich 2006), en Sudamérica estos antecedentes son escasos. No obstante, en Chile se han realizado algunos estudios en el continente antártico sobre las enfermedades que afectan a pinnipedios, siendo pesquisadas mayoritariamente enfermedades de tipo zoonóticas, como virus de influenza, las bacterias Erysipelothrix sp., Leptospira sp., Brucella sp. y Mycobacterium bovis (Blank et al. 2002).

Chile presenta una gran diversidad de mamíferos marinos, siendo el lobo marino común, Otaria flavescens (Shaw, 1800), una de las especies de mayor abundancia, en la costa chilena (Sielfeld et al. 1997, Sielfeld 1999) y en zonas cercanas de las diferentes ciudades. En Chile, a la fecha no existen registros sobre la presencia de cepas de Escherichia coli patógenas causantes de cuadros diarreicos en lobos marinos y su relación con las condiciones sanitarias del medio ambiente.

En la bahía de Iquique se vierten las aguas servidas de la ciudad, lo cual origina que las condiciones sanitarias no sean óptimas para una lobera ubicada al interior de ella. Por esta razón, en el presente trabajo se estudió la presencia de cepas patógenas de E. coli en las deposiciones de la colonia de lobos marinos en dos localidades del norte de Chile, bahía de Iquique, sitio de mucha actividad antrópica y en Punta Patache, sitio en el cual hay menor intervención humana y sin vertedores de aguas servidas. 


\section{Material y métodos}

Se analizaron 15 muestras de fecas de lobo de mar obtenidas en dos sitios donde se emplazan colonias de lobos: bahía de Iquique (20¹0S; $\left.70^{\circ} 9 \mathrm{~W}\right)$ y Punta Patache (2048; $\left.70^{\circ} 12 \mathrm{~W}\right)$. Además se obtuvieron y analizaron seis muestras de agua de mar adyacente a las loberas (bahía de Iquique, Punta Negra, centro de la bahía, Caleta Riquelme y Punta Patache). Adicionalmente, se obtuvieron tres muestras de aguas servidas, vertidas en bahía de Iquique y analizadas en su carga de contaminación fecal (NCh. 1620/1.Of84). Todas las cepas fueron aisladas en agar McConkey identificadas de acuerdo a sus propiedades bioquímicas y mantenidas a $20^{\circ} \mathrm{C}$, en una mezcla de caldo tripticasa y glicerol (50\%).

La detección de cepas patógenas de $E$. coli se realizó mediante la amplificación de los genes de virulencia asociados a las categorías diarreogénicas en estudio, de acuerdo a Stacy-Phipps et al. (1995) (Tabla 1). El templado se obtuvo mediante ebullición (Moraga et al. 2007). La amplificación se realizó en un termociclador Amplitron II, Thermolyne ${ }^{\circledR}$, de acuerdo con el siguiente programa: desnaturalización inicial a $94^{\circ} \mathrm{C}$ por $5 \mathrm{~min}, 10$ ciclos de $94^{\circ} \mathrm{C}$ por $2 \mathrm{~min}, 40^{\circ} \mathrm{C}$ por $1,5 \mathrm{~min}, 72^{\circ} \mathrm{C}$ por 1 min, luego 20 ciclos de $94^{\circ} \mathrm{C}$ por 2 min (Vidal et al. 2004). Los productos de la amplificación se detectaron mediante electroforesis en geles de agarosa al 1\%, el cual se corrió a $80 \mathrm{~V}$ por $1 \mathrm{~h}$. El producto de amplificación se visualizó en un transiluminador de luz UV (LabNet), después de teñirse con bromuro de etidio $\left(0,5 \mathrm{mg} \mathrm{mL}^{-1}\right)$.

\section{Tabla 1}

Primers usados para la amplificación de genes de virulencia de $E$. coli diarreogénicas

Primers used for amplification of virulence genes of diarrheagenic E. coli

\begin{tabular}{|c|c|c|}
\hline Gen & Secuencia partidores $\left(5^{\prime} \rightarrow 3^{\prime}\right)$ & $\begin{array}{c}\text { Tamaño del } \\
\text { producto (pb) }\end{array}$ \\
\hline \multirow[t]{2}{*}{$s t x l$} & CAG TTA ATG TGG TGG CGA AGG & 348 \\
\hline & CAC CAG ACA ATG TAA CCG CTG & \\
\hline \multirow[t]{2}{*}{ stx 2} & GCG TCA TCG TAT ACA CAG GAG C & 584 \\
\hline & GCG TCA TCG TAT ACA CAG GAG C & \\
\hline \multirow[t]{2}{*}{ eaeA } & TCA ATG CAG TTC CGT TAT CAG TT & 482 \\
\hline & GTC AAG TCC GTT ACC CCA ACC TG & \\
\hline \multirow[t]{2}{*}{ daaE } & GAA CGT TGG TTA ATG TGG GGT AA & 542 \\
\hline & TAT TCA CCG GTC GGT TAT CAG T & \\
\hline
\end{tabular}

Se realizó la genotipificación de las cepas aisladas, mediante la amplificación de secuencias intergénicas de consenso repetitivas de enterobacterias (ERIC-PCR). En este estudio se consideró como genotipo, aquellos perfiles que se agrupan en un cluster que presentan una similitud superior a 75\% (Padilla \& Ventura 2003). La amplificación se realizó en un termociclador Amplitron II, Thermolyne, de acuerdo con el siguiente programa: $95^{\circ} \mathrm{C}$ por $5 \mathrm{~min}$, cuatro ciclos de $94^{\circ} \mathrm{C}$ por $1 \mathrm{~min}, 26^{\circ} \mathrm{C}$ por $1 \mathrm{~min}, 72^{\circ} \mathrm{C}$ por $2 \mathrm{~min}, 40$ ciclos de $94^{\circ} \mathrm{C}$ por $30 \mathrm{~s}$, $46^{\circ} \mathrm{C}$ por $30 \mathrm{~s}, 72^{\circ} \mathrm{C}$ por 1 min y una extensión final por 10 min a $72^{\circ} \mathrm{C}$. Los productos de la amplificación se detectaron mediante electroforesis en geles de agarosa al $2 \%$, el cual se corrió a $60 \mathrm{~V}$ por $2 \mathrm{~h}$. Las imágenes de los geles obtenidos se guardaron como archivos TIFF. Se construyó una matriz de presencia-ausencia de bandas y se analizó mediante el programa TREECON, versión 1.3b (Universidad de Konstanz, Alemania). La construcción de los dendrogramas y el nivel de similitud entre los perfiles se calcularon mediante el coeficiente de Nei y Li (1979). El análisis de cluster de las matrices de similitud se calculó utilizando el método UPGMA (Unweighted Pair Group Method with Arithmetic mean).

\section{Resultados y discusión}

De acuerdo a los resultados del análisis de contaminación fecal (Tabla 2), los porcentajes de contaminación que se obtuvieron en Bahía Iquique, sector Punta Negra, fue de un $100 \%$ tanto para coliformes totales (CT) y coliformes fecales (CF); en tanto para Caleta Riquelme la presencia de CT fue de $100 \%$ y de un 57\% para CF, mientras que en Punta Patache los resultados fueron negativos, lo cual da cuenta de la actividad antropogénica existente en bahía de Iquique. Las aguas de bahía de Iquique presentaron altos índices de contaminación fecal, no así Punta Patache. En bahía de Iquique, el sector Punta Negra excedió los valores permitidos tanto para CT como CF, lo que concuerda con los resultados obtenidos para este sitio por Araya (2003), quien indicó la presencia de dos zonas que excedieron los valores permitidos, una de ellas en la boca del emisario de la bahía (Punta Negra) y otra en dirección al saco de la bahía.

La información sobre $E$. coli asociada a mamíferos marinos es escasa, sin embargo en Chile existen algunos antecedentes de virus, bacterias y hongos que afectan a la familia Pinnipedia, que causan enfermedades virales como virus pox y virus de la influenza y enfermedades bacterianas como Erysipelothrix insidiosa, Leptospira sp., Brucella sp. y Mycobacterium bovis, esta última aislada a partir de tejidos de lobo marino común sudamericano (Blank et al. 2002). Muchas de estas 
Tabla 2

Total de cepas de Escherichia coli analizadas por sitio de muestreo y calidad bacteriológica de las muestras de agua analizadas (NMP $100 \mathrm{~mL}^{-1}$ )

Number of strains of Escherichia coli analyzed by sampling site and bacteriological quality of water samples (MPN $100 \mathrm{~mL}^{-1}$ )

\begin{tabular}{lccc}
\hline Muestra & $\begin{array}{c}\mathrm{N}^{\circ} \text { de cepas } \\
\text { totales }\end{array}$ & $\begin{array}{c}\text { Coliformes totales } \\
\text { NMP } 100 \mathrm{~mL}^{-1}\end{array}$ & $\begin{array}{c}\text { Coliformes fecales } \\
\text { NMP } 100 \mathrm{~mL}^{-1}\end{array}$ \\
\hline Sitios contaminados & & & \\
$\quad$ Caleta Riquelme & 16 & $>1600$ & 920 \\
$\quad$ Punta Negra & 16 & $>1600$ & $>1600$ \\
$\quad$ Aguas servidas & 9 & $<2$ & $<2$ \\
Sitios no contaminados & 12 & & \\
$\quad$ Punta Patache & 12 & &
\end{tabular}

enfermedades han sido asociadas al contacto antropogénico, es decir, la presencia del hombre en estas poblaciones, muchas veces afecta la salud de estos organismos (Acevedo et al. 2003).

El análisis mediante la amplificación de los genes de virulencia asociadas a las categorías diarreogénicas de E. coli, reveló la presencia de EPEC, mediante la detección del gen eae en 18 cepas, equivalente al 38,3\% del total de las muestras analizadas, 12 cepas correspondieron a cepas aisladas desde el emisario de Punta Negra, que descarga sus aguas en la bahía. Sin embargo no se detectó la presencia del gen en las cepas provenientes de fecas de $O$. flavescens de ambas colonias analizadas.

En las cepas de $E$. coli aisladas de Bahía Iquique y de aguas servidas, se detectó la presencia el gen eae en un 100 y 77\% respectivamente, confirmando la presencia de estas cepas en las aguas de la bahía. La no detección de cepas diarreogénicas en las fecas de lobo de mar estudiadas mediante la amplificación de factores de virulencia, se debería quizás a la falta de receptores especie específicos que estarían condicionando la negatividad de los resultados, o en parte por el tiempo de exposición de las fecas a las condiciones ambientales, las cuales estarían determinando la viabilidad de los microorganismos, aunque para la realización de este estudio se muestrearon deposiciones frescas de lobo de mar. Por otra parte, se puede señalar que la presencia de cepas enteropatógenas en aguas de la bahía y no en las fecas de la colonia estudiada de Otaria flavescens, se debería a que los vertebrados, en general, presentan mecanismos de defensas e inmunidad, con los cuales los organismos en condiciones normales de salud pueden combatir una gran cantidad de microorganismos entéricos, potencialmente patógenos, presentando una inmunidad innata o adquirida, impidiendo así la invasión y proliferación de éstas (Moreno et al. 2002). Se puede señalar que no existen estudios sobre las barreras de inmunidad innata que presentan los lobos marinos, como son la acidez gástrica, motilidad intestinal, inflamación, mucosidades, etc. Teniendo solo como referencia a los mamíferos terrestres en cautiverio: ganado vacuno, porcino y ovino, en quienes se han reportado infecciones intestinales producidas por $E$. coli patógenas, es probable que su inmunidad innata no ha sido suficientemente efectiva para impedir el ingreso y proliferación de estas bacterias causando así la enfermedad (Zamora 1997).

Para el gen daa en ECAD, se obtuvo una amplificación, con un producto de 542 bp. Esta cepa se aisló a partir de fecas de lobo marino de Punta Patache. Tanto para las muestras de fecas como de agua no se detectó la presencia de los genes stx1 y stx2 de ECEH.

La detección múltiple de genes de virulencia en las cepas analizadas fue concordante con los resultados obtenidos por Cebula et al. (1995) y Vidal et al. (2004), quienes demostraron la eficacia de esta metodología en el análisis de cepas entéricas asociadas a $E$. coli diarreogénicas.

El análisis de genotipificación se efectuó por origen de las muestra y en su conjunto. Al analizar todas las cepas aisladas se observó la tendencia de agruparse por su origen de procedencia, es decir, las cepas de aguas servidas tienden a asociarse entre sí, al igual que las provenientes de las fecas de lobos así como las de agua de mar (Fig. 1). 


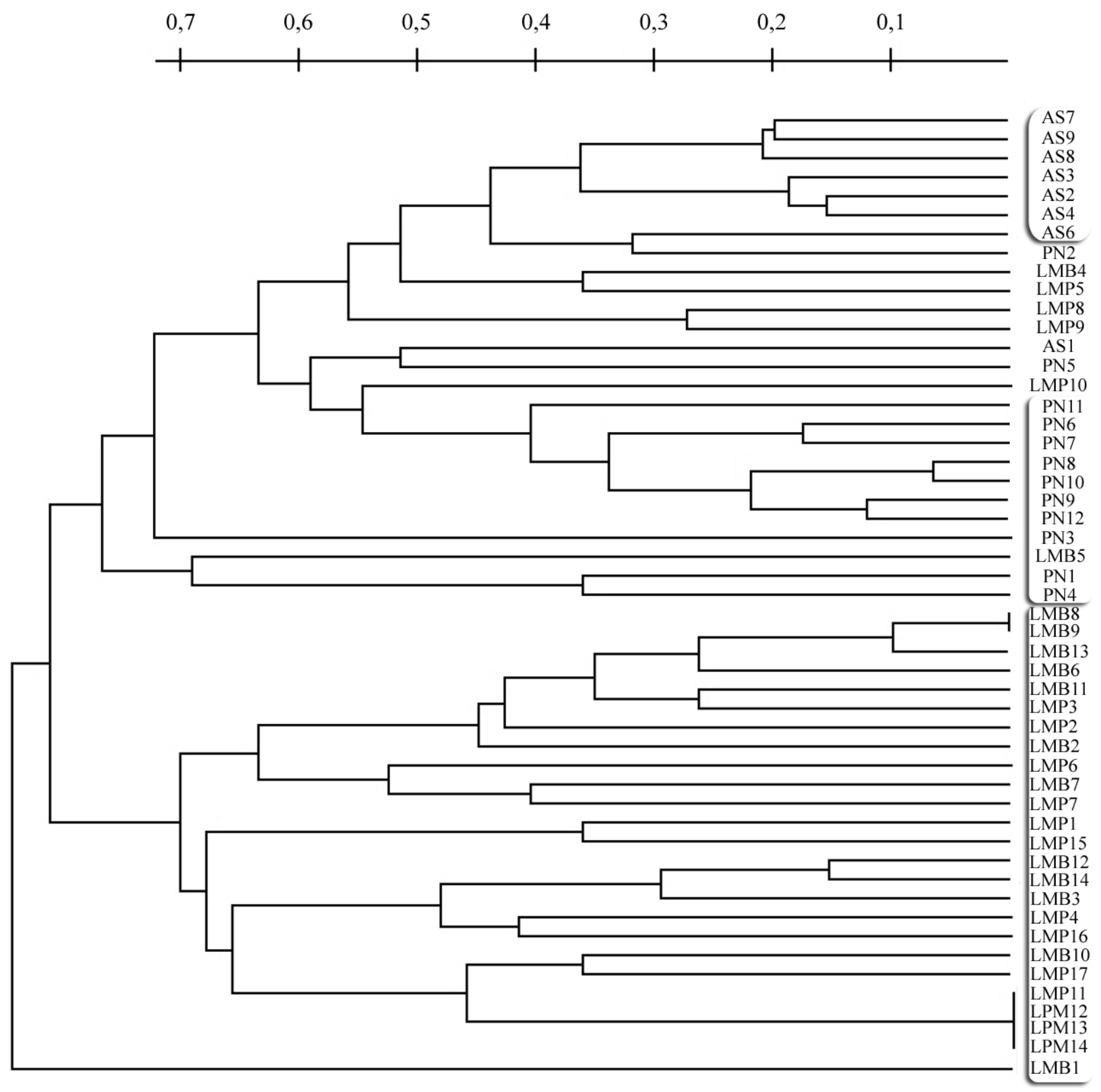

Figura 1

Análisis de cluster de los patrones de similitud basado en los perfiles de ERIC-PCR de todas las cepas analizadas en este estudio. El agrupamiento se realizó mediante media aritmética no ponderada (UPGMA). En el lado derecho, se indica el número y el origen de las cepas; LMB: lobo marino común Bahía Iquique, LMP: lobo marino común Punta Patache, PN: agua emisario Punta Negra, AS: agua servida planta tratamiento

Cluster analysis of ERIC-PCR-fingerprinting patterns of all strains analyzed in this study. Clustering was performed by an unweighted pair group method with arithmetic average (UPGMA). On the right-hand side, the strain numbers and their origin of the strains are indicated; LMB: South American sea lion at Iquique Bay, LMP South American sea lion at Punta Patache, PN: water channel Punta Negra, AS: wastewater treatment plant 
La técnica ERIC-PCR permitió determinar la diversidad clonal entre las cepas analizadas y los patrones de bandas obtenidos, que revelaron una gran variedad genética entre los aislados definiendo diferentes genotipos (Padilla \& Ventura 2003). El análisis global permitió agrupar a las cepas de acuerdo al origen de la muestra (Lueng et al. 2004). Esto concuerda con estudios realizados por Dombek et al. (2000) y Tim et al. (2003) donde se demuestra mediante otra técnica de "fingerprinter" (BOX), en cepas humanas y de animales, que tienden agruparse de acuerdo a su origen. La mayor variabilidad genética de cepas de E. coli se presentó en la población de lobo marino común de Punta Patache, lo cual es concordante con lo señalado por Souza et al. (1999) demostrando que las cepas de E. coli en poblaciones de animales salvajes presentan una extensa diversidad genética.

\section{Agradecimientos}

Los autores agradecen a la Universidad Arturo Prat por el financiamiento otorgado a través del proyecto DI 03/ 2006, que permitió la realización de este estudio.

\section{Literatura citada}

Acevedo K, H de la Cueva, M Gulland, D Aureoles, F Arellano \& F Suárez. 2003. Evidence of Leptospira interrogans infection in California sea lion pups from the Gulf of California. Journal of Wildlife Diseases 39(1): 145151.

Araya M. 2003. Contaminación de origen fecal en las principales playas del litoral de la ciudad de Iquique- Chile entre 1999-2003. Tesis de Biología Marina, Departamento de Ciencias del Mar, Universidad Arturo Prat, Iquique, 47 pp.

Blank O, P Retamal, P Abalos \& D Torres. 2002. Detección de anticuerpos Anti-Brucella en focas de Weddell (Leptonychotes weddellii) de cabo Shirref Antártica. Archivos de Medicina Veterinaria 34(1): 117-122.

Blanco J, M Blanco, JE Blanco, A Mora, MP Alonso, E González \& M Bernández. 2001. Enterobacterias características generales. Género Escherichia coli. En: Vadillo S \& E Mateos (eds). Manual de microbiología veterinaria, pp. 301-325. McGraw-Hill, Madrid.

Ceballos G \& PR Ehrlich. 2006. Global biodiversity hotspots and conservation: insights from mammal distributions. Proceedings of the National Academy of Science 103: 19374-19379.

Ceballos G, PR Ehrlich, J Soberón, I Salazar \& J-P Fay. 2005. Global mammal conservation: what must we manage? Science 309: 603-607.
Cebula T, W Payne \& P Fena. 1995. Simultaneous identification of strain of E. coli serotype O157:H7 and their shiga-like toxin type by mismatch amplification mutation assay-multiplex PCR. Journal of Clinical Microbiology 33: 248-250.

Cloete T \& N Muyima. 1997. Microbial community analysis: The key to the design of biological wasterwater treatment systems. International Association on Water Quality Technical Report 5: 1-98.

Dombek P, L Johnson, S Zimmerley \& M Sadowsky. 2000. Use of repetitive DNA sequences and the PCR to differentiate Escherichia coli isolates human and animal source. Applied and Environmental Microbiology 66: 25722577.

García J, P Jiménez, M Reche \& I Rodríguez. 2002. Microbiología clínica en fauna salvaje. Boletín Informativo de la Sociedad Española de Microbiología 33: 19-23.

Lueng K, R Mackereth, Y-C Tien \& E Topp. 2004. A comparison of AFLP and ERIC-PCR for discriminating Escherichia coli from cattle, pig and human sources. FEMS Microbiological Ecology 47: 111-119.

Moraga R, E Santander, T Arias \& F Mendez. 2007. Integrones y su relación con el fenotipo de resistencia en bacilos Gram negativos aislados en el Hospital Torres Galdames de Iquique, Chile. Revista Chilena de Infectología 24: 385-391.

Moreno A, J Gatell \& J Vila. 2002. Gastroenteritis infecciosa en el adulto inmunocompetente e inmunodeprimido. Enfermedades Infecciosas y Microbiología Clínica 8(71): 3789-3795.

Nataro J \& J Kaper. 1998. Diarrheagenic E. coli. Journal of Clinical Microbiology 11: 142-201.

Nei M \& Li WH. 1979. Mathematical model for studying genetic variation in terms of restriction endonucleases. Proceedings of the National Academic of Sciences 76: 5269-5273.

Padilla C \& G Ventura. 2003. Genotipificación de aislamientos de Bartonella bacilliformes por amplificación de elementos repetitivos mediante el uso de REP-PCR y ERIC-PCR. Revista Peruana Experimental de Salud Pública 20: 128131.

Romero J \& H Rodríguez. 1983. Niveles de contaminación coliforme en el sistema lagunar de Carmen-Machona, Tabasco. Anales del Instituto de Ciencias del Mar y Limnología 9(1): 121-126.

Sielfeld W. 1999. Estado del conocimiento sobre conservación y preservación de Otaria flavescens (Shaw, 1800) y Arctocephalus australis (Zimmermann, 1783) en las costas de Chile. Estudios Oceanológicos 18: 81-96. 
Sielfeld W, C Guerra, LR Durán, E Acuña, A Aguayo-Lobo, M Sepúlveda, F Palma, A Malinarich, G Cerda, A Bolvarán, $R$ Grau, $X$ Veloso, Y Guerra, M Vargas, $N$ Amado, $R$ Peredo \& J Galaz. 1997. Monitoreo de pesquería y censo del lobo marino común en el litoral de la I-IV Regiones. Informe Final Proyecto FIP 95-28: 1-105. [en línea] <http://www.fip.cl/prog_subprog/1995/ 9528.htm>

Souza V, M Rocha, L Sande \& L Equiarte. 1999. Genetic structure of natural populations of Escherichia coli in wild hosts on different continents. Applied and Environmental Microbiology 65(8): 3373-3383.

Stacy-Phipps S, J Mecca \& J Weiss. 1995. Multiplex PCR assay and simple preparation method for stool specimen detect enterotoxigenic $E$. coli DNA during the course of infection. Journal of Clinical Microbiology 33: 1054-1059.
Tim K, R Mackereth, Y Tien \& E Topp. 2003. A comparison of AFLP and ERIC-PCR analyses for discriminating Escherichia coli from cattle, pig and human source. FEMS Microbiology Ecology 47: 111-119.

Vidal R, M Vidal, R Lagos, M Levine \& V Prado. 2004. Multiplex PCR for diagnosis of enteric infections associated with diarrheagenic Escherichia coli. Journal of Clinical Microbiology 43(10): 1787-1789.

Zamora J, G Reinhardt, N Tadich, M Polette \& M Jaramillo. 1997. Propiedades hemaglutinantes de cepas de E. coli aisladas de corderos diarreicos y su relación con su toxicidad. Archivos de Medicina Veterinaria 29(1): 13-19.

Recibido el 03 de junio de 2009 y aceptado el 18 de diciembre de 2009 